\title{
Ultrasonic Sensor System for Ecology Observation and Timing Synchronization of ZigBee Network
}

\author{
Atsushi MORIKAWA, Hiroshi YAMAMOTO, Maki YAMAMOTO, Katsuyuki YAMAZAKI \\ Nagaoka University of Technology, 1603-1 Kamitomioka, Nagaoka, Niigata 940-2188 Japan \\ morikawa@stn.nagaokaut.ac.jp
}

\begin{abstract}
Timing synchronization is essential in case where multiple sensor nodes with ultrasonic sensors are deployed on the same place. This is because the ultrasonic sensors should be powered at an appropriate timing so as to prevent ultrasonic interference. In this paper, we propose a harmful bird attack system and a new timing synchronization method for ZigBee sensor network. In this timing synchronizing method, by concentrating the processing load to the server, the amount of calculation on each node can be minimized and is unaffected by the increase in the number of nodes. Evaluation of the proposed method has shown that it sufficiently fulfills the timing requirement for the proposed harmful birds attack system.
\end{abstract}

Keywords - Sensor Network, ZigBee, Ultrasonic Distance Sensor, Timing Synchronization, Reference Broadcast Synchronization

\section{INTRODUCTION}

Environmental and ecology observation have been performed by human beings (ecologists) with a limited set of equipment. However, a field of the observation is very severe for the ecologists to walk around freely, hence the real-time observation have not been performed. A sensor network is an emerging technology for the real-time observations, which makes it possible to automatically collect data from a large number of sensors dispersed over a vast field.

On the other hand, timing synchronization is needed in case where multiple sensor nodes such as ultrasonic sensors are deployed on the same place. This is because the ultrasonic sensors should be powered at an appropriate timing so as to prevent ultrasonic interference. However, existing timing synchronization methods [1]-[4] have those problems as they require a processing power to sensor nodes, and hence do not scale well when many and low-cost sensor nodes are to be deployed.

Therefore, in this paper, we propose a new timing synchronization method on ZigBee sensor network. In the proposed system, the amount of processing on each node is unaffected by the increase in the number of nodes, hence it can be used widely for low-cost sensor nodes. An ecology observation is an example use of the proposed method, which is also presented in this paper.

\section{RELATED WORKS AND OBJECTIVES OF THIS STUDY}

\section{A. Issues of Harmful Birds}

In recent years, the number of predation damages by harmful birds such as herons and great cormorants has been increasing. These birds have a high level of submerging, flying and breeding abilities, and eat wide variety of fishes. It was reported that estimated damage in 2007 was about 7.3 billion yen [5].

A fishway is a structure constructed for fishes to the upper stream over barriers (such as dams) as shown in Figure 1 [6]. However, many fishes are eaten there by birds because fishes should pass the narrow way, and hence become easy targets. Ascending of salmon has a critical role to keep ecosystem in a river, hence the predation issue has not only a risk of fishery damages but also a risk of ecosystem destruction.

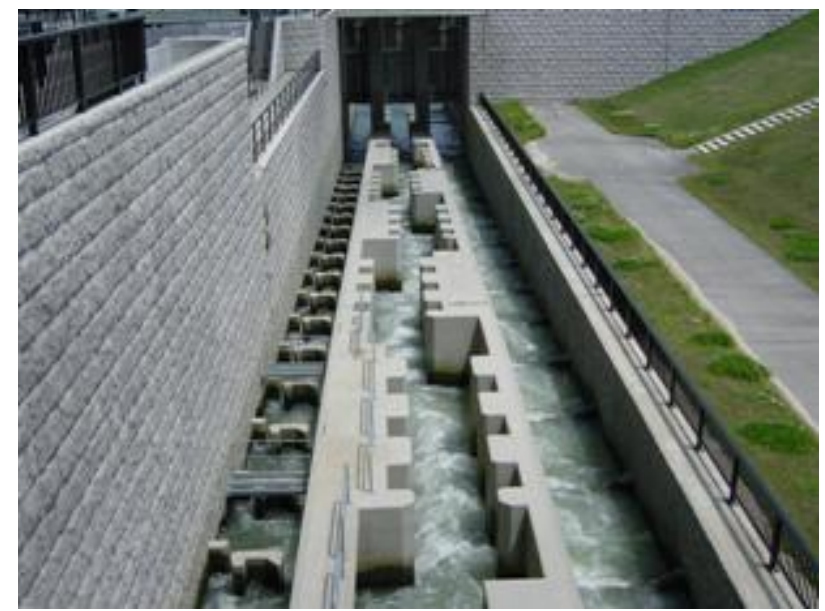

Figure 1. Fishway

Figure 2 shows a fishing weir, which is one of the traps for river fishes. The fishing weir enables to capture a lot of fishes (e.g., sweetfishes) effectively. Those captured sweetfishes are subject to cultivation for appropriately maintaining ecological environment in a river. In these fishing weirs, there are also predation problems of the fish by harmful birds. 


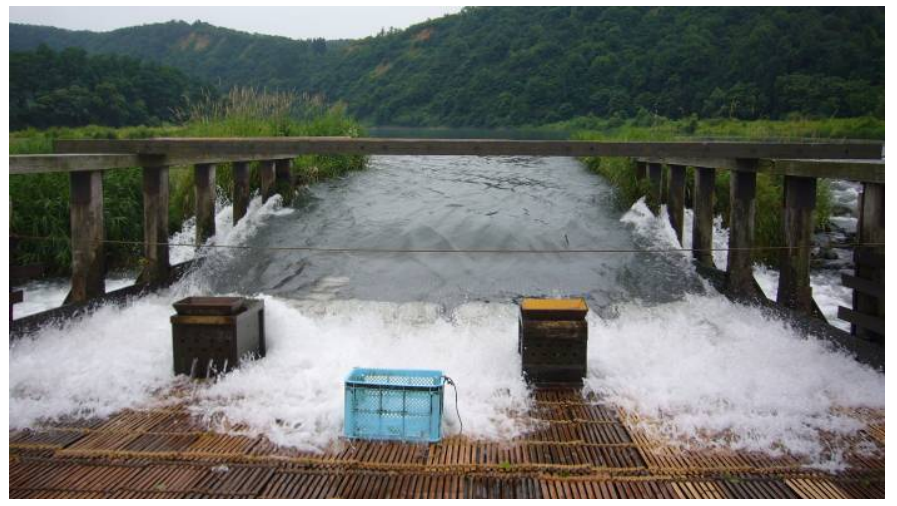

Figure 2. Fishing weir

\section{B. Use of Sensor Network}

Until now, a fishway and a fishing weir have been defended by a sound of a radio or a scarecrow. However, the birds are very clever, and become accustomed to the monotonous stimulation. Therefore, a variety of stimulations is needed in order to scare them off effectively in the long run. In this study, we propose to solve the problem by introducing a wireless sensor network that detects birds using multiple sensors (e.g., ultrasonic sensors) and scares them away by actuating various attacking devices dynamically.

\section{ZigBee Sensor Network}

A ZigBee sensor network is appropriate for ecology observation. A use of ZigBee technology was utilized for observation of birds that build a nest on a steep hill [7], [8]. In addition, cost reductions and miniaturizations of both sensors and communication modules are ensured in recent years. As a result, they enable us to deploy many sensor nodes. However, cooperation among multiple sensor nodes that have actuators or ultrasonic sensors needs a timing synchronization. Furthermore, a sensor nodes used for ZigBee sensor network is not intended for a lot of communication and calculation.

Considering these situations, we propose a new timing synchronization method that is suitable for ZigBee sensor networks.

\section{III.OVERVIEW OF THE Proposed SysteM}

\section{A. Overview of the System}

Figure 3 shows an overview of a proposed harmful bird attack system, and the devices utilized for the system are summarized in Table 1. The sensor network is constructed by sensor nodes [9], sensor arrays to detect birds, several bird attack devices and a server. The sensor array consists of ultrasonic distance sensors that are arranged in an arc shape so as to measure a wide area. The sensor node obtains sensor data from the sensor arrays, and transmits that to the server through ZigBee network. The proposed system uses three different kind of attacking devices (speakers, flashing lights and guns) so as to give various stimuli. If birds are detected by the sensors, the attacking devices drive off them.

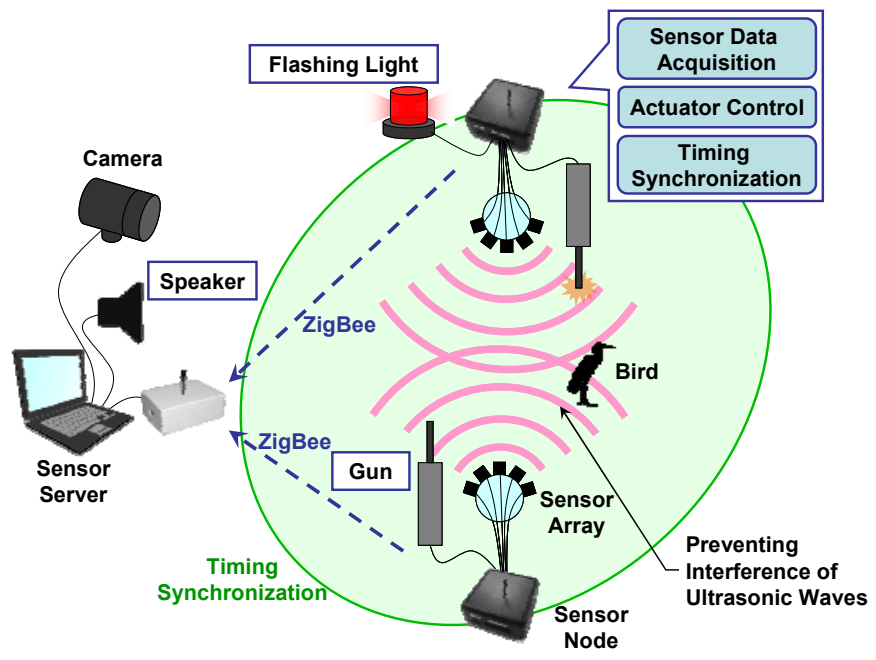

Figure 3. Overview of the harmful birds attack system

TABLE 1. Devices Constructing the Proposed System

\begin{tabular}{|c|c|l|}
\hline Role & Device & \multicolumn{1}{c|}{ Note } \\
\hline $\begin{array}{c}\text { ZigBee } \\
\text { communication }\end{array}$ & Sensor server & Storing sensed data \\
\cline { 2 - 3 } Bird detection & Sensor array & $\begin{array}{l}\text { Controlling the sensors and the } \\
\text { attacking devices } \\
\text { sensors }\end{array}$ \\
\hline \multirow{2}{*}{ Bird attacking } & Flashing light & Controlled by the sensor nodes \\
\cline { 2 - 3 } & Gun & Speaker \\
\cline { 2 - 3 } $\begin{array}{c}\text { Acquiring } \\
\text { reference data }\end{array}$ & Camera & $\begin{array}{l}\text { Installed for an experimental } \\
\text { evaluation }\end{array}$ \\
\hline
\end{tabular}

\section{B. Proposed Ultrasonic Sensor Array}

There are many studies of bird detection method using camera images [10], [11]. However, accuracy of the detection is affected by changes of brightness around the camera, and it cannot be used at night. Therefore, a sensor array that consists of several ultrasonic distance sensors is proposed as illustrated in Figure 4.

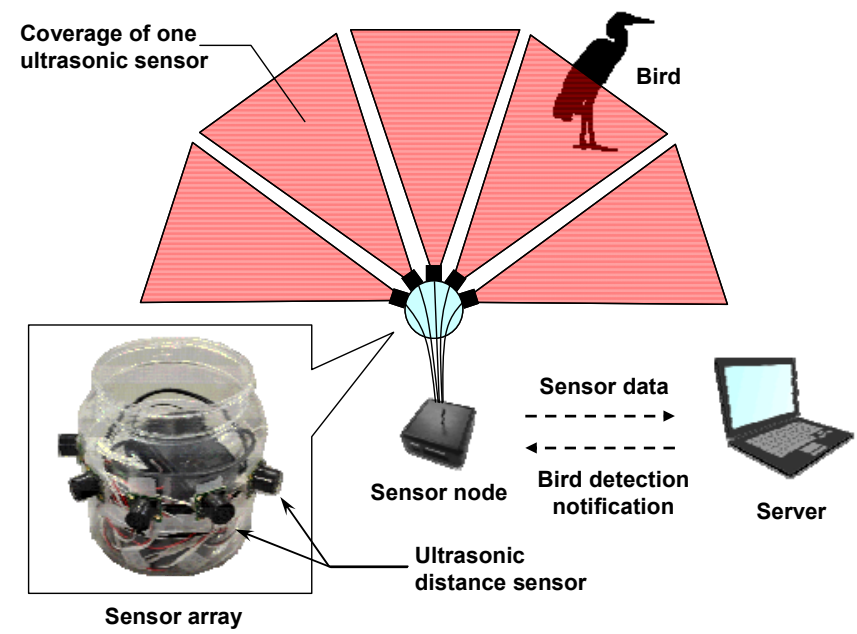

Figure 4. Bird detection method using a sensor array 
Here, when multiple ultrasonic sensors are used at the same time, they cannot get the correct results due to interference of their ultrasonic waves shown in Figure 3. Therefore, the sensor node must accurately control ON/OFF of each sensor so as to prevent the interference.

\section{IV.PROPOSED TIME SYNCHRONIZATION METHOD FOR ZIGBEE SENSOR NETWORK}

\section{A. Timing Requirement of Sensor Nodes}

When multiple sensor arrays are utilized, the interference between ultrasonic sensors must be avoided. Therefore, it is necessary to synchronize the timing between the sensor nodes and control the ultrasonic sensors so as to avoid the interference among the sensor arrays connected to different sensor nodes as illustrated in Figure 5.

The ultrasonic distance sensor used in the system is XLMaxSonar-EZL0 [12]. It generates ultrasonic waves, and a distance from an object is calculated from a time interval between generating waves and receiving the echo. The maximum range of the sensor is 1067 [cm] and the sound velocity at sea level is $340[\mathrm{~m} / \mathrm{s}]$, hence the maximum time interval for transmitting the ultrasonic is 61.9 [ms].

On the other hand, in the previous study [13], we have performed a people observation by using the sensor array. From this study, it has been found that the sensor array can accurately detect a pedestrian by operating each sensor at every 100 [ms]. Therefore, we assume that the sensor can detect a bird by the same sampling rate as the previous research. In order to operate the sensors at every 100 [ms], the synchronization error should be decreased lower than 38.1 $(100-61.9)[\mathrm{ms}]$, which becomes the requirement for the timing synchronization between sensor nodes.

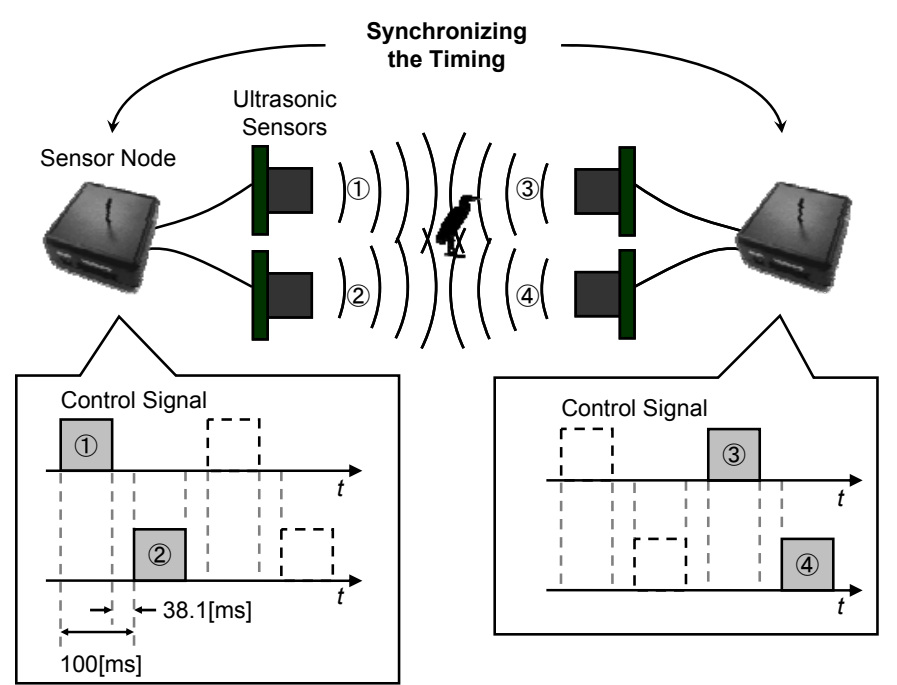

Figure 5. Timing synchronization

\section{B. Issues of Sensors in ZigBee Network}

NTP (Network Time Protocol) [1], [2] is often used as a timing synchronization method on the Internet, and synchronizes a clock of a node to the NTP server. However, the sensor network in our study does not need an absolute time, but requires synchronized timing among nodes. In addition, the NTP can not be used without connecting to the Internet, and the sensor network may be deployed in rural areas where an Internet access cannot be provided.

On the other hand, PTP (Precision Time Protocol) [14], [15] is a protocol that enables terminals to accurately synchronize with others. However, the PTP should be implemented at a hardware-level, and hence cannot be easily adapted to the sensor network.

Furthermore, GPS (Global Positioning System) [16] is also used as a timing synchronization method. The time synchronization method using the GPS takes high cost and consumes too much power because a lot of computation is needed. In addition, it cannot be used in a place where the radio wave from the satellite is blocked by the special terrain or plants. Thus, the method is not suitable for the sensor network.

In the sensor networks, RBS (Reference Broadcast Synchronization) [3], [4] is a candidate for the timing synchronization method. The procedures of the RBS are summarized as follows.

1. A server broadcasts a packet for synchronization called a reference packet.

2. Nodes receiving the packet record an arrival time of the reference packet.

3. The recorded time information is exchanged among nodes.

4. Each node calculates the time difference between the own node and a reference node (e.g., the node with the earliest clock) based on the time information, and sets the time so that the difference becomes zero.

In RBS, each node calculates correction time, hence the amount of calculation in each node increases with the increase in the number of nodes. However, many wireless sensor nodes do not have high processing power because a power saving performance often has the highest priority for an ecology observation. Thus, RBS is not appropriate for the objective.

Considering these situations, we propose a scalable timing synchronization method for ZigBee sensor network as an expansion of RBS.

\section{Procedure of Proposed Timing Synchronization Method}

The sensor nodes used in this study do not need a real time clock. Therefore, a value of a counter based on the CPU clock is a target of the synchronization. 


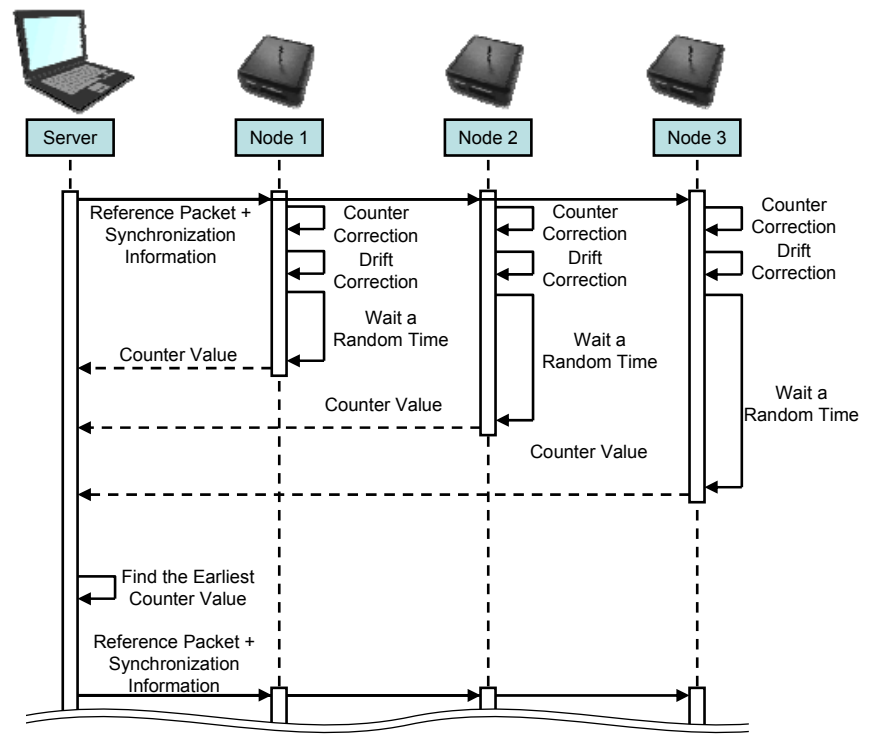

Figure 6. Sequence of the proposed synchronization method

We propose a new timing synchronization method that can reduce the computational load on each sensor node. Figure 4 represents the sequence of the proposed synchronization method. In the method, all nodes should be installed in a location where they are able to communicate directly with the server. Timing synchronization is performed as the following steps.

1. Initialization Process

- A server broadcasts a packet for synchronization called a reference packet.

- Each node obtains a counter value when receiving the reference packet.

2. Counter Correction Process

- After a random interval is passed, the node sends the value of the counter to the server.

- The server finds the earliest counter value and sends the next reference packet including the value.

- Each node obtains the counter value at the arrival time of the packet, and adjusts its counter to that of the earliest node.

In addition, a node that has a slow speed CPU clock should correct its drift. Thus, if a node corrected its counter three times continuously, it performs the drift correction process below.

3. Drift Correction Process

- The node calculates differences between its counter and the earliest node's counter per unit time as a correction value.

- The median value of the last three correction values is added to its ticking.

By concentrating the processing load to the server, the amount of calculation on a node can be reduced, and unaffected by the increase in the number of nodes.

\section{Evaluation of the Proposed Timing SYNCHRONIZATION METHOD}

\section{A. Conditions of the Experiment}

To evaluate the proposed timing synchronization method, we have implemented the proposed method to two sensor nodes and a server.

First, a synchronization interval is set. When the two nodes are not synchronized, their clocks drift $1.0[\mathrm{~ms}]$ per a second. Before the drift correction, a maximum synchronization interval $t$ is determined by the following equation.

$$
t=\frac{\delta}{2 \rho}
$$

$\delta$ is a target value of timing synchronization accuracy, and $\rho$ is a drift rate of clocks.

In the environment, the drift rate of the sensor nodes is 0.1 [\%]. In addition, the synchronization accuracy should be reduced lower than 38.1 [ms] as mentioned in Section IV. Accordingly, the synchronization should be executed at intervals of $t$ derived by Eq. (2) or shorter time interval.

$$
t=\frac{38.1}{2 \times 10^{-3}}=1.91 \times 10^{4}[\mathrm{~ms}]=19.1[\mathrm{~s}]
$$

In our evaluation, the synchronization interval is set to 15.0 [s] in order to adequately meet the timing requirement.

\section{B. Results and Discussions}

Figure 7 shows the difference of the counter values between the two nodes when they are not synchronized and when they are synchronized by the proposed method. Under the condition of the nodes without timing synchronization, the difference of the counter values is linearly increased by the error of clock. On the other hand, the difference is suppressed by synchronizing the timing.

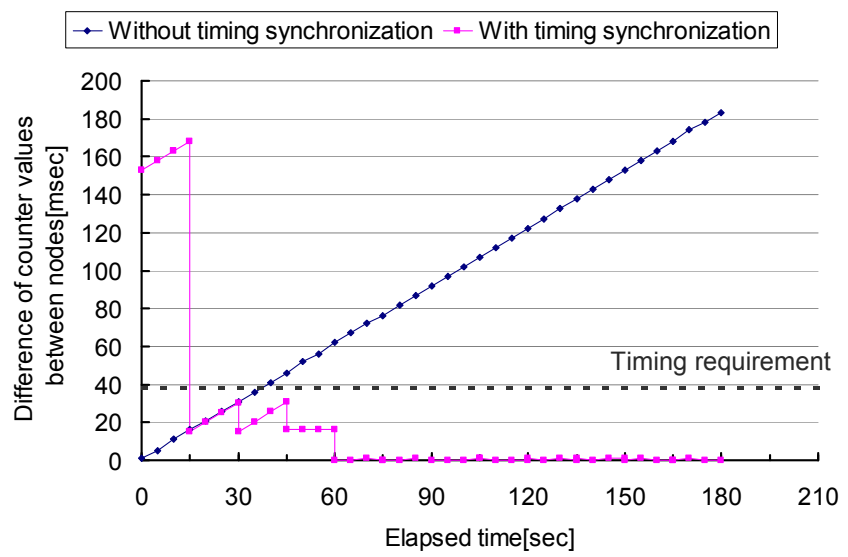

Figure 7. Difference of counter values

The timing synchronization process is clearly shown in Figure 7. First, each node receives a reference packet without a synchronization information when elapsed time is 0 [s]. Then, after 15 [s] passed, the nodes receive the next reference packet, and the delayed node corrects its counter to that of the 
earlier node. Similarly, the counter correction is performed at $30[\mathrm{~s}]$ and 45 [s]. After the process is performed three times continuously by the same node, the drift correction is processed, and hence the difference of the counter value remains static. After the fourth counter correction, it remains almost the same.

The timing synchronization is completed after the fourth counter correction at the earliest. Statistics of the difference of the counter values from that time are summarized in Table 2.

TABLE 2. STATISTICS OF THE DifFERENCE OF THE COUNTER VALUES AFTER THE FOURTH COUNTER CORRECTION

\begin{tabular}{|c|c|c|}
\hline \multicolumn{3}{|c|}{ After the fourth counter correction } \\
\hline $\begin{array}{c}\text { Maximum error } \\
{[\mathrm{ms}]}\end{array}$ & $\begin{array}{c}\text { Average } \\
{[\mathrm{ms}]}\end{array}$ & $\begin{array}{c}\text { Standard deviation } \\
{[\mathrm{ms}]}\end{array}$ \\
\hline 2 & 0.5 & 0.6 \\
\hline
\end{tabular}

The standard deviation is $0.6[\mathrm{~ms}]$, and there is a slight variation in the difference of counter values between the synchronized nodes. It is caused mainly by a buffer of a ZigBee module XBee Series 2 [17] that is mounted on the node. We have measured a time from when the module receives radio wave of a packet till when it transmits the packet as serial data. Its standard deviation is 0.84 [ms], and hence it is clear from the result that the variability is a major cause.

However, in this result, the maximum error is 2 [ms], and it is sufficiently lower than 38.1 [ms] that is the timing requirement of the proposed harmful birds attack system. Therefore, the proposed timing synchronization method has sufficient accuracy of timing synchronization for the system.

\section{VI.AN EXPERIMENT AT A FISHING WEIR}

The harmful birds attack system is being installed at a fishing weir in Nagaoka City [18] as shown in Figure 8.

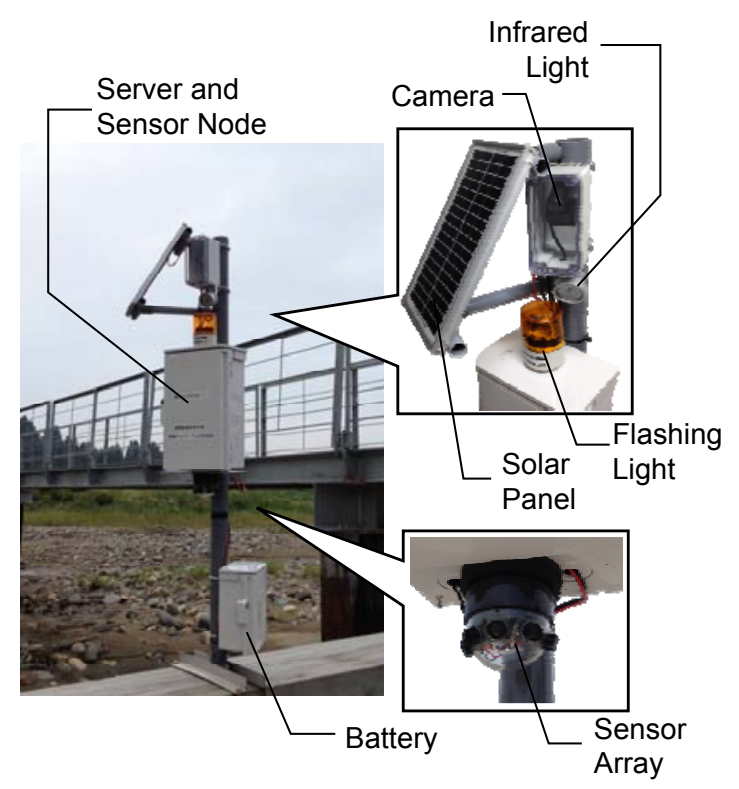

Figure 8. Installed equipment
The purpose of the experiment is to confirm that the sensor data shows the different tendency from the usual when the bird appears in the weir. Furthermore, the system has an infrared camera in order to get reference data. Moreover, a flashing light is utilized as an attacking device, and power is supplied from a battery and a solar panel to the system because the system is set up on the river.

From the experiment, a heron was captured by the camera images as shown in Figure 9. Thus, we will analyze the sensed data in order to establish a bird detection algorithm.

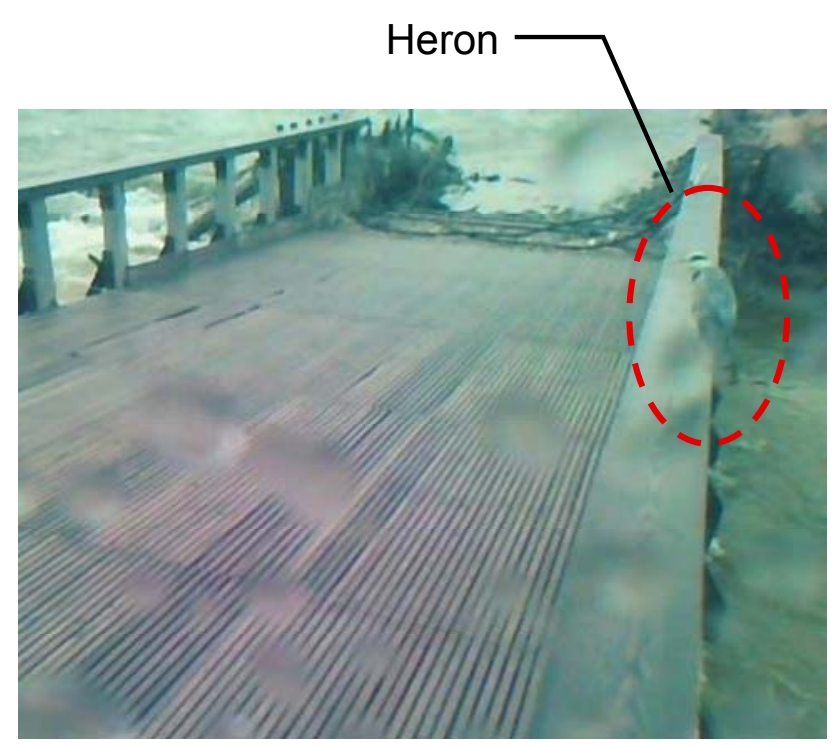

Figure 9. An example of the camera image

\section{CONCLUSIONS AND FUTURE WORKS}

This paper has proposed a harmful birds attack system. Specific features of this system are that 1) it can detect birds in wide area by using the ultrasonic sensor arrays and 2) it has a wide variety of attacking devices in order to scare clever harmful birds off.

In addition, a new timing synchronization method for ZigBee sensor network to avoid ultrasonic interference has been proposed. This method has a feature that the amount of calculation on a sensor node is small and unaffected by the increase in the nodes.

Evaluation of the proposed timing synchronization method has shown that the maximum timing error is 2 [ms] and it sufficiently fulfills the timing requirement for the proposed system.

In the future study, we'll focus on detecting and driving away harmful birds. Particularly, we will implement a control function of an ice gun [19], which is one of the attacking devices as shown in Figure 10.

This study was partly supported by MEXT/JSPS KAKENHI Grant Number 23500083. 


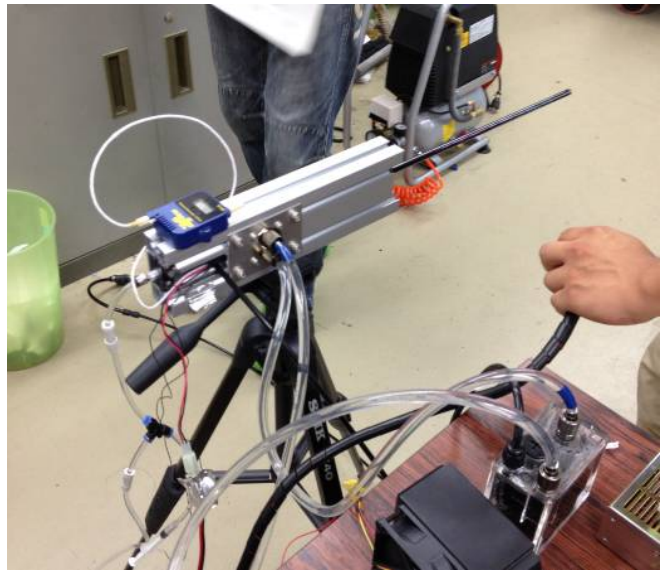

Figure 10.

An ice gun

\section{REFERENCES}

[1] RFC1129, "Internet Time Synchronization: the Network Time Protocol"

[2] RFC2030, "Simple Network Time Protocol (SNTP) Version 4 for IPv4, IPv6 and OSI"

[3] J. Elson, L. Girod and D Estrin, "Fine-grained Network Time Synchronization using Reference Broadcasts", Proc. of Operational Systems and Design Implementation, vol. 36, pp. 147-163, Dec. 2002.

[4] J. Elson and K. Romer, "Wireless Sensor Networks: A New Regime for Time Synchronization", Proc. of the First Workshop on Hot Topics in Networks (HotNets-I), October 28-29 2002.

[5] National Federation of Inlandwater Fisheries Cooperatives, "Heisei 18 Nendo Kawau Syokugai Chousa", http://www.naisuimen.or.jp/kawau/kawau18.pdf

[6] Hokuriku Regional Development Bureau Ministry of Land Infrastructure and Transport Shinanogawa River Office "Ayu No Sojo", http://www.hrr.mlit.go.jp/shinano/shinanogawa info/ayu/, Accessed December 9, 2013.

[7] S. Uchiyama, M. Yamamoto, K. Nakamura, and K. Yamazaki, "Sensor Network for Observation of Seabirds in Awashima Island", Proc. of IEEE Information Networking (ICOIN), 2011 International Conference on, pp.64-67, January 2011

[8] H. Kurazono, H. Yamamoto, M. Yamamoto, K. Nakamura, and K Yamazaki, "RFID and ZigBee Sensor Network for Ecology Observation of Seabirds", Advanced Communication Technology (ICACT), $201315^{\text {th }}$ International Conference on, pp. 211-215, January 2013

[9] S. Minomi, H. Yamamoto, K. Nakamura, and K. Yamazaki, "A Study of Digital Signage Attention Estimation System with Ultrasonic Distance Sensor", The Institute of Electronics, Information and Communication Engineers Trans., Vol.J95-D, No.5, pp.1193-1195, May 2012.

[10] Y. Kuboyama, N. Mita, and T. Yoshioka, "Bird Detection in Images with Optical Flow", Forum on Information Technology vol. 6(3), pp. 25-26, August 2007

[11] W. W. Verstraeten, B. Vermeulen, J. Stuckens, S. Lhermitte, D. Van der Zande, and et al., "Webcams for bird detection and monitoring: A demonstration study", Sensors 2010, 10, 3480-3503.

[12] MaxBotix, http://www.maxbotix.com/

[13] A. Morikawa, H. Yamamoto, K. Nakamura, and K. Yamazaki, "Evaluation of Ultrasonic Sensor Array for Crowd Observation System", Proc. of the Institute of Electronics, Information and Communication Engineers Shin-etsu Session, P2-1, p. 192, October 2012

[14] IEEE Standard 1588, "IEEE Standard for a Precision Clock Synchronization Protocol for Networked Measurement and Control Systems", November 2002.

[15] M. Hiyama, M. Kaneko, and K.Yamazaki, "Implementation of Precision Clock Synchronization Protocol to IEEE802.11", The
Institute of Electronics, Information and Communication Engineers Tech. Rep., vol.109, IA2009-86, February, 2010.

[16] D. E. Comer, "Computer Networks and Internets", Prentice Hall, pp.287-288, 2008 .

[17] Digi International, http://www.digi.com

[18] Echigo Kawaguchi Yanaba, http://kawaguchiyanaba.s1.bindsite.jp/

[19] T. Yamane, and S. Kamimura, "Development of Anti-Vermin Ice Gun -Part 2 ballistic of Ice bullet and point of impact-", Proc. of the Japanese Society of Snow and Ice and Japan Society for Snow Engineering Joint Conference, p. 119, September, 2010.

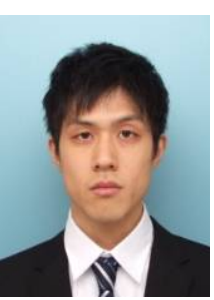

Atsushi Morikawa received B.E. degree from Nagaoka University of Technology in '12. He is currently a graduate school student in Nagaoka University of Technology. His research interests include computer networks and sensor networks.

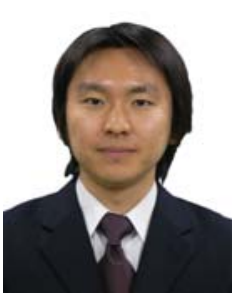

Hiroshi Yamamoto received M.E. and D.E. degrees from Kyushu Institute of Technology, Iizuka, Japan in '03 and '06, respectively. From April '06 to March '10, he worked at FUJITSU LABORATORIES LTD. Kawasaki, Japan. Since April '10, he has been an Assistant Professor in the Department of Electrical Engineering, Nagaoka University of Technology. His research interests include computer networks, distributed applications, and networked services. He is a member of the IEEE.

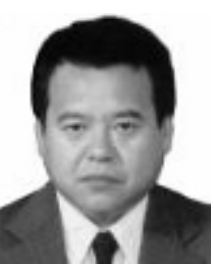

Katsuyuki Yamazaki received B.E. and D.E degrees from the University of Electro-communications and Kyushu Institute of Technology in '80 and '01, respectively. At KDD Co. Ltd., he had been engaged in $\mathrm{R} \& \mathrm{D}$ and international standardization of ISDN, S.S. No.7, ATM networks, L2 networks, IP networks, mobile and ubiquitous networks, etc., and was responsible for R\&D strategy of KDDI R\&D Labs. He is currently a Professor of Nagaoka University of Technology. 